\title{
Prevalence and Antibiogram of Stenotrophomonas maltophilia in a Tertiary Care Hospital at Trivandrum, Kerala, India
}

\author{
Sunil Kumar D Chavan ${ }^{1}$, K.H. Harshan ${ }^{2 *}$, T. Vineetha ${ }^{1}$, Chandrasekaran Kavita ${ }^{3}$ \\ ${ }^{1}$ Department of Microbiology, GMC, Kannur, Kerala, India \\ ${ }^{2}$ Department of Microbiology, MZMC, Adoor, Kerala, India \\ ${ }^{3}$ Periodontist and Implantologist, Kannur, Kerala, India \\ *Corresponding author
}

\section{Keywords}

Stenotrophomonas maltophilia, antibiogram, antimicrobial susceptibility, antibiotic resistance

Article Info

Accepted:

05 January 2020

Available Online:

10 February 2020

A B S T R A C T

Stenotrophomonas maltophilia causes opportunistic infections and is an emerging opportunistic pathogen. The rate of infections due to these bacteria has increased in recent years and so is their resistance to antimicrobials. Thus, the present study was conducted with an objective to identify Stenotrophomonas isolation from various clinical samples and to study its antimicrobial sensitivity/ resistance pattern which will guide clinicians in prophylactic antibiotic therapy. A total of 400 clinical samples were collected from patients admitted in ICU and other wards of the hospital. All samples were inoculated on to Blood Agar (BA) and MacConkey Agar (MA) plates under strict aseptic conditions, followed by incubation at $37^{\circ} \mathrm{C}$ for $24-48$ hours under aerobic conditions. Antimicrobial susceptibility testing was performed by Kirby Bauer disc diffusion method on Mueller Hinton agar. Out of 400 samples, $48.57 \%$ sputum samples, $34.38 \%$ of pus sample, $14.28 \%$ blood ssmples and $2.85 \%$ of other samples were positive for isolates.

\section{Introduction}

Originally classified as Pseudomonas maltophilia, Stenotrophomonas maltophilia is an obligate aerobe and a ubiquitous organism. It causes opportunistic infections and is an emerging opportunistic pathogen. It is the third most commonly encountered non- fermenter in clinical laboratory next to Pseudomonas and Acinetobacter. It is an important nosocomial pathogen associated with substantial morbidity and Mortality rate of $43 \%$ especially in immunosuppressed patients, patient in intensive care unit, and pulmonary source of the isolate. (1) 
The most common site for recovery of S.maltophilia is the respiratory tract. It is frequently isolated from patients with ventilator support in ICU. It produces proteolytic enzymes, deoxyribonucleases, ribonucleases, hemolysins, hyaluronidase and mucinase etc. which contribute to its severity in immunosuppressed patients.

The rate of infections caused by S.maltophilia is increased in recent years and are being isolated from wound infections, bacteremia, pneumonia, endocarditis, urinary tract infections, meningitis and peritonitis. S.maltophilia is oxidase negative, motile, catalase positive, indole negative, citrate variable, urease negative,lysine and DNAse positive. $(2,3)$

S.maltophilia is susceptible to colistin and polymyxin. The antibiotic susceptibility pattern can be a clue to the identification of S.maltophilia. The most active agents are trimethoprim sulphamethoxazole, colistin and quinolones. Like other nonfermenters it is intrinsically resistant to many common antibiotics like aminoglycosides, carbapenams and many betalactam agents. Development of resistance in these organism is multifactorial.

Factors involved are-mutations in genes encoding porins, efflux pump mechanisms, penicillin binding proteins, chromosomal beta lactamases. Success of antimicrobial therapy depends on the appropriateness of the choice of antibiotics that should be used on the basis of prior knowledge of the susceptibility pattern of the agent. $(4,5)$

Epidemiological studies of clinical $S$. maltophilia isolates have shown genetic diversity, probably associated with selection of naturally present $S$. maltophilia from among other bacteria by antibiotic pressure. However, cross-infections between patients, transmitted by healthcare workers, have also been reported. For this reason, detection of antibiotic resistance patterns and typing of $S$. maltophilia isolates is significant in the context of hospital infection control. Itis a readily available commensal of importance, found in water, soil, sewage and frequently on plant or within plant rhizosphere.(6)

The bacteria explore the depression of immune systems to cause infection, though they have also been implicated in infection of immunocompetent subjects. They are therefore important considering their infectivity and the morbidity they initiate, which range from nosocomial to community acquired infections. It propagates in moist environments (water, medical equipment, soil and sewage) and colonizes medical devices.

Dialysed patients are an ideal target for infections: they are immunosuppressed because of uraemia, old age, malnutrition, comorbidities and the increased use of artificial accesses such as prosthetic grafts, central venous or peritoneal catheters.(7)

The present study was conducted with an objective to identify Stenotrophomonas isolation from various clinical samples and to study its antimicrobial sensitivity/ resistance pattern. This study is also directed to guide the clinicians for prophylactic antimicrobial therapy.

\section{Materials and Methods}

The study was conducted at Shree Gokulam Medical College and Research centre, Trivendrum from January 2016 to December 2016. A total of 400 clinical samples were collected from patients admitted in ICU and various wards of the hospital of depending upon the clinical diagnosis of respective patients. 
Out of these 35 isolates of Stenotrophomonas was isolated. These included: pus, blood, sputum and other samples. All samples were collected and processed as per standard microbiological guidelines. Samples were inoculated on to Blood Agar (BA) and MacConkey Agar (MA) plates under strict aseptic conditions and plates were incubated at $37^{\circ} \mathrm{C}$ for $24-48$ hours under aerobic conditions. (8)

All isolates that showed non-lactose fermenting colonies on MA and those which grew only on BA and not on MA were subjected to Gram staining and all gramnegative bacilli/cocci/coccobacilli obtained were then subjected to triple sugar iron test. The bacterial isolates which produced alkaline/acid (K/A) reaction and acid/acid (A/A) reaction were excluded.

Isolates which produced an alkaline/alkaline $(\mathrm{K} / \mathrm{K})$ reaction were provisionally identified as non-fermenters and were included in this study and subjected to identification upto genus/species level by a battery of biochemical tests.8 Oxidative/Fermentative $(\mathrm{O} / \mathrm{F})$ test for glucose, lactose, sucrose, mannitol and xylose, oxidase test, motility test, nitrate reduction test, lysine and ornithine decarboxylase test, arginine dihydrolase test, gelatin liquefaction test, urease test, indole production test, citrate utilization test, growth at $42^{\circ} \mathrm{C}$ and $44^{\circ} \mathrm{C}$.

Antimicrobial susceptibility testing was performed by Kirby Bauer disc diffusion method on Mueller Hinton agar as per CLSI guidelines using commercially available discs. (9, 10, 11) Following antimicrobial discs were used: ceftazidime $(30 \mu \mathrm{g})$, cefepime $(30 \mu \mathrm{g})$, piperacillin-tazobactum $\quad(100 \mu \mathrm{g} / 10$ $\mu \mathrm{g})$, aztreonam $(30 \mu \mathrm{g})$, imipenem $(10 \mu \mathrm{g})$, meropenem $(10 \mu \mathrm{g})$, gentamicin $(10 \mu \mathrm{g})$, amikacin (30 $\mu \mathrm{g})$ netilmicin $(30 \mu \mathrm{g})$, ciprofloxacin $(5 \mu \mathrm{g})$, norfloxacin $(30 \mu \mathrm{g}$; for urinary isolates), polymyxin B (300 units) and colistin $(10 \mu \mathrm{g})$.

Plates were incubated at $37^{\circ} \mathrm{C}$ for $18-24$ hours and results were interpreted according to zone sizes mentioned in the CLSI guidelines. (12)All dehydrated media and antibiotic discs were procured from HiMedia Labs, Mumbai, India.

Statistical analysis was done by descriptive statistics using percentages and ratios method. Sensitivity was performed using control strains of Staphylococcus aureus ATCC25923, E. coli ATCC25922, and Pseudomonas ATCC 27853.

\section{Results and Discussion}

Between Jan 2016 to Dec 2016, 35 patients with underlying haematological or oncological disease were diagnosed with $S$. maltophilia bacteraemia. Of these, 15 had Diabetes mellitus, 10 had COPD, 5 patients were on ventilator and 5 patients were immunocompromised. The demographical data and patients' sample wise distribution are listed in Table 1 and 2.

Table.1 Gender Distribution $(n=35)$

\begin{tabular}{|l|l|l|}
\hline Sex & Number & Percentage (\%) \\
\hline Male & 23 & 65.72 \\
\hline Female & 12 & 34.28 \\
\hline
\end{tabular}


Table.2 Sample wise distribution $(\mathrm{n}=35)$

\begin{tabular}{|l|l|l|l|}
\hline Sample & Size & Isolates & Percentage (\%) \\
\hline Sputum & 120 & 17 & 48.57 \\
\hline Pus & 202 & 12 & 34.28 \\
\hline Blood & 45 & 5 & 14.28 \\
\hline Others & 33 & 1 & 2.85 \\
\hline Total & 400 & 35 & 100 \\
\hline
\end{tabular}

Table.3 Comorbidity conditions ( $\mathrm{n}=35$ )

\begin{tabular}{|l|l|l|}
\hline $\begin{array}{l}\text { Underlying } \\
\text { conditions }\end{array}$ & Number & Percentage (\%) \\
\hline COPD & 10 & 28.57 \\
\hline Ventilator & 5 & 14.28 \\
\hline Diabetes mellitus & 15 & 42.85 \\
\hline Immunocompromised & 5 & 14.28 \\
\hline
\end{tabular}

Maximum isolates were obtained from sputum and pus samples followed by blood and others. Out of 400 samples, $48.57 \%$ sputum samples, $34.38 \%$ of pus sample, $14.28 \%$ blood samples and $2.85 \%$ of other samples were positive for isolates.

Table 3 and 4 represents the results of the comorbid conditions and antibiogram conducted on the isolates. S. maltophilia was also found to be multidrug resistant pathogen showing resistance to various groups of antibiotics. All isolates of S. maltophilia were resistant towards Amikacin and Gentamicin, Sensitive to Ceftazidime, CefoperazoneSulbactum, Piperacillin-Tazobactum and to Cotrimoxazole.

Out of 35 patients, 10 patients (28.57\%) suffered from COPD, 15 patients were diabetic, 5 patients $(14.28 \%)$ were on ventilator and 5 patients (14.28\%) were immunocompromised.

Of all the isolates, $100 \%$ resistance was observed towards Amikacin and Gentamicin. $100 \%$ sensitivity was observed towards Ceftazidime, $96 \%$ sensitivity towards Cafoperazone-sulbactam combination, 94\% sensitivity towards Piperacilin-tazobactam combination and $93 \%$ sensitivity towards Cotrimoxazole.

Non-fermenters were usually considered as commensals or contaminants in the past but have now emerged as important health care pathogens. These organisms are associated with life threatening infections such assepticaemia, pneumonia, UTI, meningitis, surgical site infections, ventilator associated pneumonia, osteomyelitis etc. and resistance to antimicrobials have resulted in difficulty in treatment of infections caused by these bacteria. (13)

NFGNB are intrinsically resistant to various antimicrobials and are known to produce extended spectrum betalactamases (ESBL's) and metallobetalactamases (MBL's). 
Table.4 Antibiogram $(n=35)$

\begin{tabular}{|l|c|c|}
\hline Antibiotics & Sensitive & Resistant \\
\hline Ceftazidime & $\mathbf{1 0 0}$ & $\mathbf{0}$ \\
\hline Cotrimoxazole & 93 & $\mathbf{7}$ \\
\hline $\begin{array}{l}\text { Cefoperazone- } \\
\text { sulbactam }\end{array}$ & 96 & 4 \\
\hline $\begin{array}{l}\text { Piperacillin- } \\
\text { tazobactam }\end{array}$ & 94 & 6 \\
\hline Amikacin & & \\
\hline Gentamicin & $\mathbf{0}$ & $\mathbf{1 0 0}$ \\
\hline
\end{tabular}

The recovery rate of this bacterium appears to be increasing with time compared to when the bacteria was initially discovered. (14)

Fluoroquinolone and polymycin B, both of which showed good activities against the $S$. maltophilia isolates, are usually the antibiotics of choice in the treatment of infections by the bacteria. The activities of these antibiotics against the bacteria have been similarly reported by Gales et al., and Tripodi et al., $(15,16)$ However, it is known that trimethoprim-sulfamethoxazole is the drug of therapeutic choice against $S$. maltophilia infections ; but several reports have shown that the prevalence of $S$. maltophilia strains that are resistant to trimethoprim-sulfamethoxazole are increasing. (17)

S. maltophilia causes infections mainly in hospitals and is a particular risk for debilitated patients. This organism is ubiquitous in the environment and in the hospital setting. Since it is able to grow in many different media in the presence of most antimicrobial agents, $S$. maltophilia is isolated with increasing frequency as a nosocomial pathogen.

The annual isolation rate per 10 000patient discharges rose from 7.1 in 1981 to 14.1 in 1984 at a university hospital in the USA. (18) A widespread study between 1997 and 2001, including data from Asia-Pacific, Europe and
America, showed that S. maltophilia was the third most frequently isolated nonfermentative bacterium, following $P$. aeruginosa and Acinetobacter, with a rate of isolation from clinical specimens of $8 \%$. (19)

As described above, the isolation frequency of S. maltophilia increased during the period of the present study (8.5\%), but further investigations are needed to clarify the underlying reasons for this increase.

In the present study, S. maltophilia isisolated most often from respiratory specimens (sputum), pus and blood. Valdezate et al., described about 105 S. maltophilia isolates obtained between 1995 and 1998, 79 of which were from the respiratory tract and 19 from blood. (20).

Isolation of S. maltophilia from polymicrobial cultures may be related to a true infection, and is an important consideration in determining initial treatment, since b-lactamases leaking from S. maltophilia cells can facilitate the survival of b-lactam-susceptible microorganisms $(21,22)$.

The many risk-factors that predispose to the development of $S$. maltophilia infection include prolonged hospitalisation, especially in ICUs, consumption of broad-spectrum antibiotics, malignancy, immune suppression, and a breakdown in mucocutaneous defence 
barriers (e.g., following catheterisation, artificial implants, tracheostomy, or peritoneal dialysis). (23) Most of the patients (85.4\%) in the present study had underlying diseases, including $14.6 \%$ who had malignant diseases. These results are in accordance with previously published data. S. maltophilia is resistant to a wide spectrum of antimicrobial agents.

In a worldwide surveillance study that included 1488 isolates obtained between 1997 and 2001, resistance to the antimicrobial agents tested was $>50 \%$, withthe exception of co-trimoxazole (5\%), gatifloxacin (5\%), levofloxacin (6\%), ticarcillin-clavulanate (14\%) and ceftazidime (34\%). (24) Similarly, the present study found resistance rates of $>$ $60 \%$ for all antimicrobial agents except cotrimoxazole.

When an isolate is identified as $S$. maltophilia, cotrimoxazole, ticarcillinclavulanate, doxycycline, minocycline and the newer quinolones, such asofloxacin, levofloxacin, sparfloxacin and moxifloxacin, may be possible options for treatment. (21)

Although the NCCLS suggests the use of dilution methods for testing antimicrobial susceptibilities of $S$. maltophilia, the correlation between in-vitro resistance and the clinical response is unknown. $(25,26)$

The incubation time and temperature for susceptibility testing remain controversial, with an increase in incubation time influencing the resistance rates of $S$. maltophilia forco-trimoxazole, ciprofloxacin, b-lactams and aminoglycosides. According to the susceptibility of the bacterium, a prolonged combination therapy of endovenous aminoglycosides plus levofloxacin or ceftazidime or trimethoprim/ sulfamethoxazole, and a locked-in instillation of gentamicin or ceftazidime, is safe, with an excellent outcome for both the patient and the catheter. $(27,28)$

S. maltophilia is an emergent pathogen in dialysis units, causing infection in peritoneal and haemodialysis patients. As it is a natural inhabitant in most environments inside and outside the hospital setting, it can induce infections in immunosuppressed subjects.

From the present study it can be concluded that maximum isolates of Stenotrophomonas maltophilia can be obtained from sputum and pus of affected patients.

It has also been demonstrated from the present study that S.maltophilia is multidrug resistant to various groups of anitbiotics. In the present study, the antibiogram conducted showed that all isolates of S.maltophilia were resistant towards most commonly used penicillin Amikacin and Gentamicin. At the same time, they were sensitive to other individual and combination antimicrobials such as Ceftazidime, CefoperazoneSulbactum, Piperacillin-Tazobactum and to Cotrimoxazole.

The treatment of $S$. maltophilia-related infection could be cumbersome because of several bacterial and host characteristics. Antibiotic therapy, if well conducted, shall be able to cure the infection in most cases; in a few cases, it preserves patient safety and dialysis access patency too.

\section{References}

Kaur A, Gill AK, Singh S.Prevalence and antibiogram of nonfermenting gram negative bacilli isolates obtained from various clinical samples in a tertiary care hospital, Bathinda, Punjab, India. Int J Res Med Sci. 2018 Apr;6(4):12281234.

Ebara H, Hagiya H, Haruki Y, Kondo E, Otsuka F. Clinical Characteristics of 
Stenotrophomonas

maltophilia

Bacteremia: A Regional Report and a Review of a Japanese Case Series. Intern Med. 2017;56(2):137142.

Denton M, Kerr KG. Microbiological and clinical aspects of infection associated with Stenotrophomonas

maltophilia. Clin

Microbiol

Rev. 1998;11:57-80.

Krueger TS, Clark EA, Nix DE. In vitro susceptibility of Stenotrophomonas maltophilia to various antimicrobial combinations. Diagn Microbiol Infect Dis. 2001;41:71-78.

Nicodemo AC, Araujo MRE, Ruiz AS, Gales AC. In vitro susceptibility of Stenotrophomonas

maltophilia isolates: Comparison of disc diffusion, Etest and agar dilution methods. J.

Antimicrob

Chemother. 2004;53:604-608.

Looney WJ. Role of Stenotrophomonas maltophilia in hospital-acquired infection. $\mathrm{Br} \mathrm{J}$ Biomed Sci. 2005 ;62(3):145-54.

De Mauri A, Torreggiani M, Chiarinotti D, Andreoni S, Molinari G, De Leo M. Stenotrophomonas maltophilia: an emerging pathogen in dialysis units. J Med Microbiol. $2014 \quad \mathrm{Nov} ; 63(\mathrm{Pt}$ 11):1407-10.

Mahdi O, Eklund B, Fisher N. Laboratory culture and maintenance of Stenotrophomonas maltophilia. Curr Protoc Microbiol. 2014;32:Unit-6F.

Bauer, A. W., W. M. M. Kirby, J. C. Sherris, and M. Turck. 1966. Antibiotic susceptibility testing by a standardized single disk method. Am. J. Clin. Pathol. 36:493-496.

Clinical Laboratory Standards Institute. 2006. Performance standards for antimicrobial disk susceptibility tests; Approved standard-9th ed. CLSI document M2A9. 26:1. Clinical Laboratory Standards
Institute, Wayne, PA.

Kirby, W. M. M., G. M. Yoshihara, K. S. Sundsted, and J. H. Warren. 1957. Clinical usefulness of a single disc method for antibiotic sensitivity testing. Antibiotics Annu. 1956-1957:892.

Chung HS, Hong SG, Lee Y, Kim M, Yong $\mathrm{D}$, Seong Sk et al., Antimicrobial Susceptibility of Stenotrophomonas maltophilia Isolates from a Korean Tertiary Care Hospital. Yonsei Med J 2012; 53(2): 439-441.

Malini A, Deepa E, Gokul B, Prasad S. Nonfermenting gram-negative bacilli infections in a tertiary care hospital in kolar, karnataka. J Lab Physicians. 2009;1(2):62-66.

Baruah FK, Hussain AN, Kausalya, Grover RK. Antibiotic resistance profile of nonfermenting Gram-negative bacilli isolated from the blood cultures of cancer patients. J Glob Infect Dis. 2015;7(1):46-47.

Gales AC, Jones RN, Forward KR, Linares J, Sader HS, Verhoef J. Emerging importance of multidrug-resistant Acinetobacter species and Stenotrophomonas maltophilia as pathogens in seriously ill patients: geographic patterns, epidemiological features, and trends in the SENTRY Antimicrobial Surveillance Program (1997-1999). Clin Infect Dis. 2001;32 Suppl 2:S104-13.

Tripodi MF, Andreana A, Sarnataro G, Ragone E, Adinolfi LE, Utili R. Comparative activities of isepamicin, amikacin, cefepime, and ciprofloxacin alone or in combination with other antibiotics against Stenotrophomonas maltophilia. Eur J Clin Microbiol Infect Dis. 2001;20(1):73-5.

Al-Jasser AM. Stenotrophomonas maltophilia resistant to trimethoprimsulfamethoxazole: an increasing problem. Ann Clin Microbiol 
Antimicrob. 2006;5:23.

Gülmez D, Hasçelik G. Stenotrophomonas maltophilia: antimicrobial resistance and molecular typing of an emerging pathogen in a Turkish university hospital. Clinical Microbiology and Infection 2005; 11(11): 880-6.

Chang YT, Lin CY, Chen YH, Hsueh PR. Update on infections caused by Stenotrophomonas maltophilia with particular attention to resistance mechanisms and therapeutic options. Front Microbiol. 2015;6:893.

Valdezate S, Vindel A, Loza E, Baquero F, Cantón R. Antimicrobial susceptibilities of unique Stenotrophomonas maltophilia clinical strains. Antimicrob Agents Chemother. 2001;45(5):15811584.

Brooke JS. Stenotrophomonas maltophilia: an emerging global opportunistic pathogen. Clin Microbiol Rev. 2012;25(1):2-41.

Denton M, Kerr KG. Microbiological and clinical aspects of infection associated with Stenotrophomonas maltophilia. Clin Microbiol Rev. 1998;11(1):57-80.

Crispino M, Boccia MC, Bagattini M, Villari P, Triassi M, Zarrilli R. Molecular epidemiology of Stenotrophomonas maltophilia in a university hospital.J Hosp Infect. 2002; 52(2): 88-92.

Morrison AJ, Hoffmann KK, Wenzel RP. Associated mortality and clinical characteristics of nosocomial Pseudomonas maltophilia in a university hospital. J Clin Microbiol 1986; 24: 52-55.

Tatman-Otkun M, Gürcan S, Ozer B, Aydoslu B, Bukavaz S. The antimicrobial susceptibility of Stenotrophomonas maltophilia isolates using three different methods and their genetic relatedness. BMC Microbiol. 2005;5:24. Published 2005 May 9. doi:10.1186/1471-2180-5-24.

A. C. Nicodemo, M. R. E. Araujo, A. S. Ruiz, A. C. Gales, In vitro susceptibility of Stenotrophomonas maltophilia isolates: comparison of disc diffusion, Etest and agar dilution methods, Journal of Antimicrobial Chemotherapy, Volume 53, Issue 4, April 2004, Pages 604-608.

Samonis, G., Koutsounaki, E., Karageorgopoulos, D.E. et al., Empirical therapy with ceftazidime combined with levofloxacin or oncedaily amikacin for febrile neutropenia in patients with neoplasia: a prospective comparative study. Eur J Clin Microbiol Infect Dis 31, 1389-1398 (2012).

Tamma PD, Cosgrove SE, Maragakis LL. Combination therapy for treatment of infections with gram-negative bacteria. Clin Microbiol Rev. 2012;25(3):450-470. doi:10.1128/CMR.05041-11.

\section{How to cite this article:}

Sunil Kumar D Chavan, Harshan K H*, Vineetha T, Chandrasekaran Kavita. 2020. Prevalence and Antibiogram of Stenotrophomonas maltophilia in a Tertiary Care Hospital at Trivandrum, Kerala, India. Int.J.Curr.Microbiol.App.Sci. 9(02): 295-302. doi: https://doi.org/10.20546/ijcmas.2020.902.037 